\title{
Des indicateurs à l'action : vulnérabilité alimentaire et situation nutritionnelle en milieu rural sahélien burkinabé
}

Paru dans la revue Canadian Journal of African Studies, Toronto, Vol. 40, n 3, pp. 443-461.

\author{
Pierre JANIN $^{1}$ et Yves MARTIN-PREVEL $^{2}$ \\ ${ }^{1}$ UR 106 « Nutrition, Alimentation et Sociétés » \\ Iedes - Université de Paris I \\ 45 bis, avenue de la Belle Gabrielle \\ 94736 NOGENT SUR MARNE \\ FRANCE \\ Pierre.Janin@ird.fr \\ Pierre.Janin@neuf.fr \\ Tél. : 0033143947247 \\ ${ }^{2}$ UR 106 « Nutrition, Alimentation et Sociétés » \\ Centre IRD de Ouagadougou \\ 01 BP 182 Ouagadougou 01 \\ BURKINA FASO \\ Yves.Martin-Prevel@ird.bf \\ Tél. : 0022650306737 \\ Fax : 0022650310385
}

\section{Résumé}

Depuis les crises alimentaires des années 1970 et 1980, les pays sahéliens ont exploré diverses stratégies pour gérer le risque d'insécurité alimentaire. Cependant, leurs approches intègrent souvent mal les déterminants complexes de la vulnérabilité alimentaire et de la malnutrition.

C'est pourquoi, l'IRD et l'Université de Ouagadougou ont mis en place, entre 2001 et 2003, un dispositif d'enquêtes rurales coordonnées de terrain, dans l'Est du Burkina Faso, pour d'explorer les relations entre risques apparents spatialisés, vulnérabilité alimentaire réelle et situation nutritionnelle des individus.

Les analyses ont montré que le niveau de risque alimentaire apparent, évalué d'après les contraintes de gestion du milieu, est loin de correspondre avec les disponibilités alimentaires réelles et à l'état nutritionnel des individus. Ainsi, les niveaux de malnutrition sont les plus faibles dans la zone de risque maximale. Ceci suggère que, confrontés à des déficits céréaliers importants et récurrents, les ménages ont développé des stratégies d'adaptation et d'anticipation, permettant de gommer partiellement les aléas saisonniers.

Mots-clefs : vulnérabilité alimentaire, soudure, déficit énergétique, Burkina Faso, milieu rural. 


\section{Introduction}

Depuis les graves crises alimentaires des années 1970 et 1980, de nombreux organismes institutionnels ou privés (FEWS, PAM, FAO, USAID), travaillant à différentes échelles spatiales, ont développé plusieurs méthodes d'investigation en milieu rural afin d'améliorer les stratégies actuelles de lutte contre l'insécurité alimentaire transitoire (stockage de sécurité, aide alimentaire). Dans cette optique toujours, le suivi satellitaire des cultures, les prévisions pluviométriques ou les relevés des prix céréaliers fournissent des éléments avancés, utiles à la prise de décision. Leurs objectifs prioritaires - qui se recouvrent partiellement - visent à hiérarchiser les espaces en fonction du niveau de risque, afin d'identifier les groupes de population les plus vulnérables pour permettre une meilleure allocation de l'aide alimentaire (Jaspers and Shoham 1999 ; Clay, Molla et Habtewold 1999).

Néanmoins, ces informations instantanées permettent rarement d'apprécier le niveau réel des disponibilités alimentaires céréalières, de même que la capacité de résilience des communautés villageoises et des ménages ruraux. De même, le ciblage des zones d'intervention éventuelle et l'identification des catégories de population les plus exposées à l'insécurité alimentaire (Egg et Gabas 1997) restent difficiles à mettre en oeuvre compte tenu de la variabilité des situations interannuelles et de la dispersion géographique de l'habitat rural.

Et lorsque des efforts sont faits pour prendre en compte des facteurs d'ordre plus structurel, socio-démographique ou bio-climatique, le risque est alors de survaloriser certains indicateurs ponctuels (revenus monétaires annuels par exemple) au détriment des comportements des acteurs et de la dynamique d'ensemble du système alimentaire.

Qui plus est, le repli de l'État et l'avènement du marché conduisent de fait à une gestion de plus en plus décentralisée - familiale et communautaire - de l'insécurité alimentaire. Or, même dans un contexte de précarité monétaire et de disette récurrente, les modes de gestion des ressources - greniers et animaux - comme les recours développés en période de soudure ${ }^{1}$ (Janin 2004) revêtent une importance stratégique dont les conséquences peuvent être appréhendées d'un point de vue géographique comme nutritionnel. D'où l'importance d'une approche susceptible de mettre en évidence la capacité réelle des familles et des communautés villageoises à gérer le risque d'insécurité alimentaire et, dans la mesure du possible, à l'anticiper.

Tout ceci impose, au préalable, l'établissement d'un premier diagnostic des risques apparents à l'échelle des «petits espaces ruraux », sur la base de données secondaires, complété par la collecte d'indicateurs instantanés de gestion des ressources alimentaires à l'échelle villageoise. Ont été plus particulièrement pris en compte tous les repères, immédiatement observables ou résultant de déclarations, de déséquilibre dans le temps et dans le paysage, indiquant qu'une crise alimentaire plus grave que d'habitude peut intervenir et que les réponses communautaires sont insuffisantes ou inappropriées. La caractérisation à des échelles «désagrégées » (ménage et individu) des disponibilités alimentaires et de leurs conséquences nutritionnelles suppose, en revanche, la mise en place d'enquêtes plus importantes - par la taille des échantillons et la multiplicité des acteurs - et plus longues afin de tenir compte les effets de la redistribution sociale et de leurs variations saisonnières (Eele 1994). L'ensemble de cette démarche accorde donc très logiquement une place centrale au

\footnotetext{
${ }^{1}$ Exprimée par l'intervalle temporel entre la disparition des réserves et la prochaine récolte céréalière et par le déficit entre besoins et disponibilités alimentaires.
} 
concept de vulnérabilité ${ }^{2}$. Une des dernières difficultés réside dans le passage d'une analyse circonstanciée des risques à l'identification de propositions réalistes pour l'action.

C'est sur la base de ce constat que s'appuie la recherche menée conjointement par des géographes et des nutritionnistes de l'IRD, dans une province sahélo-soudanienne de l'Est du Burkina Faso. Un de ses objectifs prioritaires était d'explorer la nature des relations existantes - causalités, concordances et disparités - entre d'une part, les risques apparents, perçus comme tels par les décideurs et vécus par les enquêtés (aridité, enclavement, dégradation des potentialités), les déterminants réels de la vulnérabilité des ménages d'autre part, essentiellement abordés par l'analyse des modes de gestion de la soudure alimentaire, et enfin l'état nutritionnel des individus.

\section{La trame première des perceptions et des contraintes héritées}

Le choix de la province sahélo-soudanienne de la Gnagna, qui couvre environ $8.600 \mathrm{~km}^{2}$ dans l'Est du Burkina Faso, répond à plusieurs critères tant géographiques que nutritionnels. La documentation scientifique préalable disponible sur ce petit espace sahélo-soudanien est peu abondante et insiste particulièrement sur les contraintes, handicaps, retards dont il serait frappé tout en pointant les défis que les communautés et les développeurs devront relever. $\mathrm{Au}$ point de donner naissance à des perceptions très négatives, voire répulsives.

Cette province correspond à un espace de transition longtemps très enclavé (certains décideurs ont ainsi coutume de parler «d'angle mort du Burkina »), hors des grands axes de communication, et même relativement marginal par rapport au cœur historique du Gourma, dont le pôle de commandement traditionnel et administratif est Fada N'Gourma. La principale piste rurale permanente qui désenclave cette province n'a été achevée qu'en 1985. En période d'hivernage, de nombreux villages sont temporairement inaccessibles en raison d'un dense réseau hydrographique secondaire à très faible pente. Par ailleurs, une augmentation sensible des écoulements de surface a été mesurée dans l'ensemble de la partie orientale du Burkina et s'explique essentiellement par une dégradation du couvert végétal sous l'impact des défrichements agricoles et pastoraux.

Sa partie septentrionale possède les caractéristiques des milieux sahéliens dégradés tandis que les empreintes paysagères de la moitié méridionale évoquent le domaine soudanien. Comme dans l'ensemble des provinces septentrionales du Burkina, le climat de la Gnagna est caractérisé par une forte variabilité pluviométrique, aussi bien intra- qu'interannuelle et une diminution progressive du volume d'eau précipitée depuis la décennie 1960, même si les dernières années connaissent une amélioration sensible. Les relevés pluviométriques des quatre dernières décennies ont également permis de confirmer l'existence d'un gradient latitudinal d'aridité et d'instabilité. Ainsi l'indice d'aridité (rapport entre l'année la plus sèche et l'année la plus humide) est toujours supérieur à deux tandis qu'un différentiel moyen d'environ $100 \mathrm{~mm}$ se maintient entre les parties septentrionale et méridionale $(550-650 \mathrm{~mm})$.

Longtemps faiblement peuplé, cet espace agropastoral connaît, depuis les années 1980, un renforcement de la pression anthropique sur les ressources (pâturage, bois, bas-fonds, sols cultivables) en liaison avec le phénomène de colonisation agricole spontanée issue des terroirs saturés du Plateau Central mossi et avec l'arrivée d'un nombre croissant d'éleveurs semi-

\footnotetext{
2 «Par vulnérabilité alimentaire, on considère ici la sensibilité structurelle et conjoncturelle des individus, des familles, des communautés et des régions à l'insécurité alimentaire. Cette dernière dépend d'une exposition plus ou moins grande aux aléas et aux risques qui la commandent, comme d'une moindre capacité à mobiliser les ressources physiologiques, économiques, sociales ou éco-géographiques de proximité pour y faire face » (Courade 2001 et $a l$.).
} 
nomades peuls chassés par les crises climatiques. Désormais, on y observe des phénomènes plus marqués de concurrence spatiale entre communautés ${ }^{3}$ et des situations ponctuelles de saturation foncière. Toutefois, les relations inter-ethniques villageoises restent encore fortement marquées par des liens de complémentarités socio-économiques ${ }^{4}$. En 1998, la densité rurale moyenne atteignait $40 \mathrm{hab} / \mathrm{km}^{2}$ avec de fortes disparités intra-zonales ce qui, compte tenu du caractère extensif des systèmes productifs, correspond à un seuil démographique à ne pas dépasser. C'est pourquoi la production agricole est caractérisée par une grande variabilité. Ainsi le bilan céréalier régional est bien souvent déficitaire même si des excédents sont comptabilisés certaines années. En ce sens, la Gnagna semble représentative des espaces sahéliens soumis au risque récurrent de disette alimentaire (Gado 1993).

Du point de vue alimentaire, la province est caractérisée par un régime très monotone, basé essentiellement sur les céréales, avec de très faibles apports en lipides, protéines et micro-nutriments (Sawadogo 2002). Sur le plan nutritionnel, des études parcellaires font état de forts taux de malnutrition protéino-énergétique, d'anémie et de goître, mais il n'existe pas, avant nos enquêtes, de chiffres fiables à l'échelle de l'ensemble de la province. La Gnagna est également caractérisée par une mortalité infanto-juvénile très élevée, des niveaux d'hygiène très bas, un faible accès à l'eau potable et un sous-équipement en infrastructures sanitaires jusque dans les années 1990, même si un effort d'investissement important a été récemment consenti. Qui plus est, leur accessibilité reste souvent aléatoire compte tenu du mauvais état des pistes rurales et du faible nombre de transports collectifs.

Enfin, cette province est également l'une des plus enclavées et des moins alphabétisées du Burkina qui se situe, par ailleurs, déjà parmi les pays les plus mal classés. Selon les statistiques du Ministère de l'Enseignement de base et de l'Alphabétisation de masse, dans la Gnagna en 1993-94, le taux moyen de scolarisation des garçons ne dépassait pas 14,6\% pour les garçons et $5,9 \%$ pour les filles, contre $40,4 \%$ et de $27,0 \%$ pour la moyenne nationale. Pour les enfants de 7 à 12 ans, la Gnagna avait également le plus faible taux de scolarisation avec $10,3 \%$ contre une moyenne nationale de $33,8 \%$. Ces chiffres ne sont pas neutres dans la mesure où plusieurs études ont démontré le rôle joué par le niveau d'éducation féminine dans la prévalence de la malnutrition des enfants (UNICEF 1999).

L'ensemble de la documentation secondaire recueillie reste parcellaire, disparate et parfois daté. Cette dernière est également grossière puisqu'elle offre souvent des informations agrégées à l'échelle de la province ou, au contraire, très ponctuelles et localisées, sans fournir d'indications réelles sur les problèmes alimentaires et des situations nutritionnelles.

\section{Entre recherche et expertise : les indicateurs spatiaux de risque}

La première étape de la recherche proprement dite a consisté à recueillir, confronter et spatialiser les différents indicateurs de risques. La notion de risque a été considérée dans son sens le plus géographique, comme l'ensemble de chocs extérieurs déstabilisants, susceptibles de remettre en cause certains équilibres (au sens systémique du terme), auxquels sont exposés les ménages ruraux et contre lesquels il est difficile de se prémunir durablement et efficacement (Eldin 1989).

\footnotetext{
${ }^{3}$ Le fonds de peuplement gourmantché reste majoritaire (75\%), loin devant les communautés peul (17\%) et mossi (5\%).

${ }^{4}$ Confiage des bœufs gourmantché aux éleveurs peul en échange d'un droit de culture et de dons de céréales en période de soudure.
} 


\section{Carte 1 : niveaux de risques apparents}
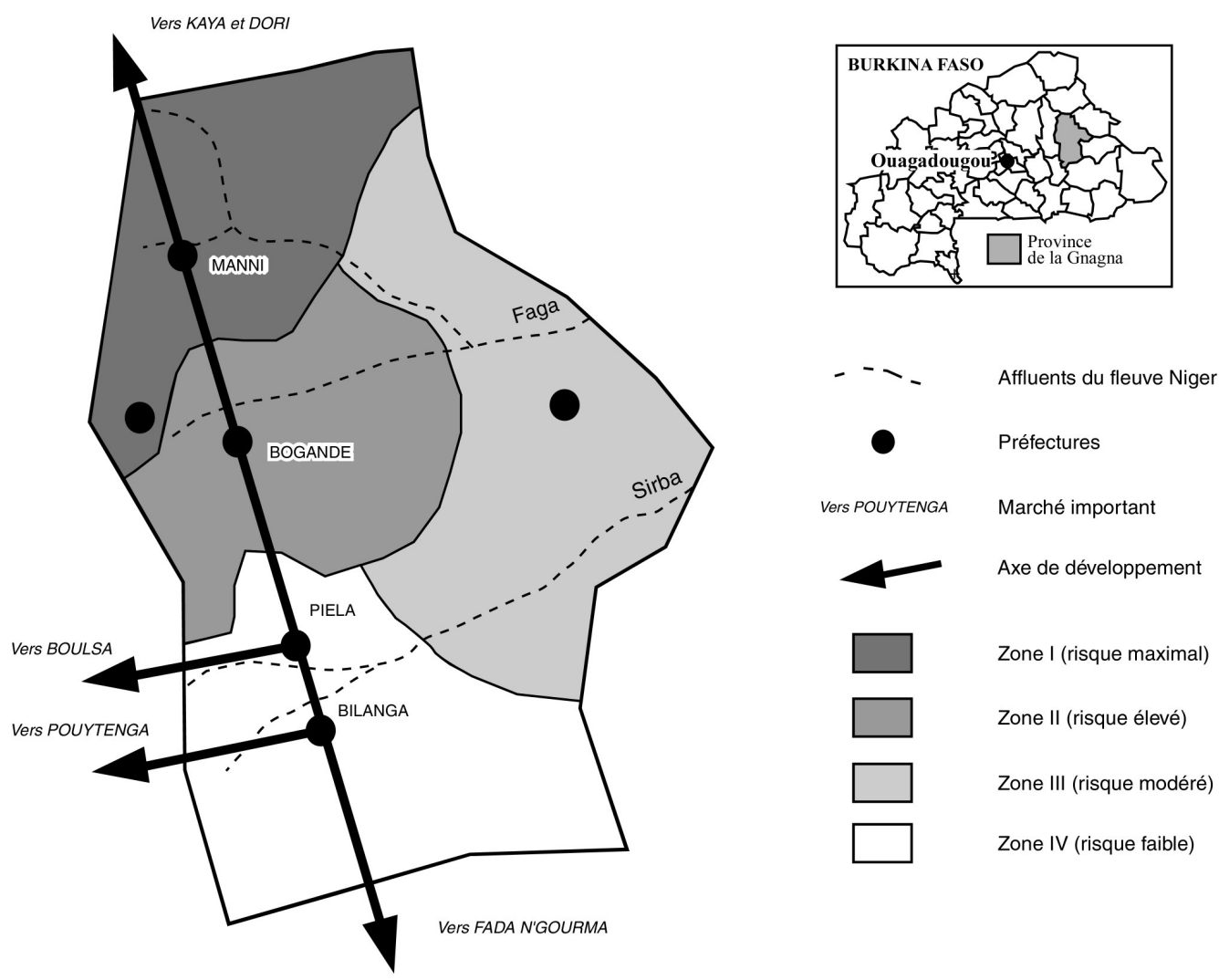

Zone I Zone II Zone III Zone IV

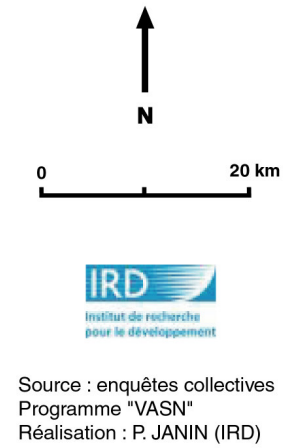

Au cours des années 2000 et 2001, l'effort de collecte de données secondaires a permis d'affiner les informations disponibles dans quelques domaines particuliers : éducation de base, pluviométrie, pauvreté, prix céréaliers sur les marchés. Néanmoins, l'ensemble des ces éléments fragmentaires n'était pas de nature à permettre de caractériser le risque d'insécurité alimentaire à "grande échelle». C'est pourquoi, une enquête rapide a été mise sur pied, sous forme de discussion avec une «sélection » de personnes-ressources ${ }^{5}$ dans l'ensemble des 276 villages de la province. Le questionnaire d'enquête de 241 questions semi-fermées a été testé au printemps 2000 avant d'être appliqué au cours de l'hivernage 2000. Des indicateurs de crises alimentaires et de contraintes géographiques (enclavement), de potentialités et de ressources mobilisables (arbres utiles, eau, types d'élevage), de gestion du milieu (densités

\footnotetext{
${ }^{5}$ Chefs de village, délégués administratifs, représentants des communautés de migrants, responsables des comités villageois, villageois.
} 
rurales, saturation foncière, déboisement) ont été recueillis au cours d'entretiens semi-dirigés collectifs dans chaque village. Nous avons donc associé des indicateurs structurels avec des éléments plus conjoncturels (Watts et Bohle 1993).

Les indicateurs les plus pertinents pour une analyse des risques ont fait l'objet d'une cartographie sous Mapinfo et Adobe Illustrator (Ouedraogo 2001) qui possède certaines similitudes avec les méthodes de cartographie spatiale de la vulnérabilité proposée par certains systèmes d'information (Moseley et Logan 2001, PAM 2002, CILSS 2000). Pour chacun d'entre eux, plusieurs niveaux d'intensité du phénomène observé ont été retenus. Par association, ils ont permis d'aboutir à une hiérarchisation synthétique des risques apparents en quatre sous-ensembles géographiques (cf. carte) qui seront conservés pour l'ensemble des analyses ultérieures, de disponibilités alimentaires et de situations nutritionnelles (Janin et Martin-Prével 2003). C'est donc une démarche essentiellement géographique qui a prévalu à ce stade de la recherche, sans chercher à délimiter des strates statistiques (Jayne et al. 2001), afin de comparer les situations économiques et alimentaires de certaines catégories d'acteurs (Dilley et Boudreau 2001).

- La première zone (de risque maximal) correspond au cœur densifié de l'espace d'étude $\left(>80 \mathrm{hab} / \mathrm{km}^{2}\right)$. Les terroirs sont bien stabilisés et en voie de saturation foncière en raison de l'installation progressive de migrants agricoles mossi. Le taux annuel de croissance naturel de la population y est, certes, élevé (autour de 3\%) mais reste nettement en deçà des autres zones. La pression pour l'accès et l'usage des ressources (terre, bois et eau) y est forte, même si la présence de plusieurs retenues d'eau a permis le développement de nouvelles activités agricoles (maraîchage, pêche, riziculture). Les sols y sont peu fertiles et fortement dégradés en raison de la disparition progressive de la jachère et de la coupe accélérée de bois de chauffe destiné à la vente. Différents types d'élevage sont pratiqués par les communautés en présence (peule, gourmantché et mossi) et les conflits de voisinage sont plus marqués. Le caractère sahélien du climat y est plus affirmé comme l'indique l'indice d'aridité plus élevé $(2,8)$. Les villages sont peu enclavés tandis que les relations d'échange marchand, plus développées, permettent de dégager des ressources monétaires supplémentaires (embouche animale, petit commerce). Cette zone apparaît cependant comme la plus exposé au risque de disette : la soudure alimentaire y est plus longue et plus fréquente tandis que l'accessibilité aux aliments y est plus aléatoire compte-tenu de la grande volatilité des prix céréaliers au cours de l'année agricole.

- La deuxième zone limitrophe (de risque aggravé) occupe une plus vaste superficie. Si les types de risques et les contraintes ne diffèrent pas sensiblement avec ceux de la zone précédente ils ne revêtent pas, en revanche, la même intensité : la soudure alimentaire est moins récurrente et plus courte. Les densités rurales sont moins élevées (40-60 hab/km²) mais restent supérieures à la moyenne provinciale de 1998. L'accès à la terre reste encore possible pour des allochtones même si la multiplication des hameaux de culture constitue un indicateur patent de saturation progressive. Toutefois, une forte majorité d'exploitants parvient à maintenir une période de jachère entre les rotations culturales. La variabilité intra-annuelle des précipitations s'estompe quelque peu (indice $<2,5$ ) tout en restant plus élevée que dans le secteur méridional sahélo-soudanien. L'enclavement géographique ne touche pas l'ensemble des villages enquêtés mais s'impose comme une contrainte saisonnière. 
- Dans les deux dernières zones, la compétition pour l'accès et l'usage de la ressource foncière ou ligneuse ne revêt pas la même intensité. Des potentialités non négligeables (terre, bois, eau) perdurent étant donné la charge démographique réduite (densités $<20 \mathrm{hab} / \mathrm{km}^{2}$ ). Toutefois, le rythme annuel de croissance démographique est nettement supérieur aux autres zones (6\% contre 3\%). En contrepartie, les activités traditionnelles d'élevage, transhumant ou non, sont plus développées compte tenu de la présence accrue d'agro-pasteurs peuls, plus ou moins sédentarisés. L'indice d'aridité décroît (pour éviter la répétition) (proche de 2) en raison d'une pluviométrie sensiblement supérieure : $650 \mathrm{~mm}$ annuels moyens depuis 1960 contre $550 \mathrm{~mm}$ dans la première zone. De ce fait, la variabilité inter-annuelle des productions se réduit sensiblement: les déficits céréaliers sont à la fois moins importants et moins fréquents. Certaines années, des excédents sont même comptabilisés.

Ce type de diagnostic territorial est relativement aisé à mettre en œuvre et apporte des éléments intéressants d'information sur les disparités et les discontinuités spatiales au sein de cette province. En outre, il associe la collecte d'indicateurs conjoncturels et structurels, répondant ainsi à une préoccupation majeure des intervenants. Il peut, de ce fait, se révéler pertinent pour l'aide à la décision dans certains domaines (projet régional de développement, plan de gestion intégrée des ressources, ciblage d'actions de développement). Néanmoins, il possède des limites intrinsèques à l'échelle d'observation et d'analyse villageoise. En effet, la localisation spatiale des villages est rendue parfois difficile par la forte dispersion spatiale de l'habitat rural et l'absence de centralité. De même, il tend à homogénéiser les situations, parfois très diverses au sein d'un même village : par exemple, les écarts spatiaux (hameaux de culture, campements peuls) ne sont pas pris en considération au risque de l'être également dans d'éventuels projets de développement. Plus problématique pour une intervention dans le domaine alimentaire et nutritionnel, le niveau réel des disponibilités alimentaires familiales a peu de chances d'être connu de même que la dynamique d'ensemble du système alimentaire régional (flux de produits, variation des prix). En effet, il s'appuie sur un ensemble de déclarations ponctuelles qui ne sont pas nécessairement mises en relation avec d'autres facteurs de risque individuel. Ainsi, cette spatialisation cartographique n'intègre pas certains phénomènes majeurs en milieu rural sahélien tels que l'effet de la saisonnalité, la succession des crises (Reardon and Matlon 1989), l'inégale vulnérabilité des familles et des individus.

\section{Risques apparents et insécurité alimentaire réelle}

Forts de ces limites inhérentes au dispositif, les chercheurs du projet «Vulnérabilité alimentaire et sécurité nutritionnelle » associant l'IRD et l'Université de Ouagadougou ont alors mis en place, en 2001 et 2002, un ensemble d'enquêtes coordonnées et approfondies, à différentes échelles (concession, ménage, individu), auprès d'un échantillon aléatoire de 180 communautés familiales (cours collectives), réparties dans 30 villages, représentant environ 600 ménages et 5600 individus. Des passages répétés ont permis de recueillir des informations socio-démographiques complètes, des indicateurs du niveau économique (y compris une estimation des revenus par individu) et des conditions de vie (hygiène, accès à l'eau potable), des informations sur la gestion de la soudure alimentaire en 2001 et 2002 (anticipation, durée, stratégies de recours), une estimation des disponibilités céréalières. Enfin, en janvier-mars 2002, des mesures anthropométriques de tous les individus ont été réalisées selon des procédures standardisées (WHO 1995). 
L'évaluation des disponibilités alimentaires présentes sur l'exploitation a été conduite selon plusieurs méthodes. La première a été réalisée sur la base des déclarations d'utilisation de quelque 1400 greniers céréaliers individuels et collectifs, en fin d'année civile (après remplissage des greniers familiaux) précédant les mesures anthropométriques. Les achats céréaliers effectués au cours de l'année 2001 d'observation ont également été comptabilisés ; ils ont été convertis en équivalent-yorouba ${ }^{6}$ sur la base de 40 yorouba par sac. Les données ainsi obtenues ont ensuite converties en jours de ration quotidienne moyenne (PAM 1999) en tenant compte des importantes variations saisonnières de la ration préparée au sein du groupe de consommation. Cette évaluation a, dans un deuxième temps, été confrontée avec le volume réel des céréales stockées dans l'ensemble des greniers qui ont tous été mesurés : ce volume a, par la suite, été converti en kilos équivalent-céréales après application d'un coefficient tenant compte de la qualité et du type de grain.

Nous avons délibérément opté pour une approche non restrictive du concept d'insécurité alimentaire. C'est pourquoi, les dotations céréalières disponibles sur l'exploitation ont été systématiquement confrontées avec certains éléments instantanés révélateurs de soudure - la capacité à anticiper les situations de déficit par des achats céréaliers précoces par exemple.

La hiérarchisation spatiale selon le niveau de risque a été reconduite pour l'analyse des données d'enquête auprès des ménages. La période d'observation de la soudure (2001) correspond à une année de disette alimentaire pour les ménages ruraux enquêtés : les récoltes y ont été médiocres en raison d'un déficit pluviométrique en milieu de l'hivernage 2000. Ceci explique la durée moyenne exceptionnelle de la soudure et sa prévalence très élevée $(90 \%$ des ménages sont concernés), avec une répartition atypique puisqu'elle dépassait 190 jours pour la moitié des ménages enquêtés.

\begin{tabular}{|c|c|c|c|c|c|}
\hline \multicolumn{6}{|l|}{ Tableau 1} \\
\hline \multicolumn{6}{|c|}{$\begin{array}{l}\text { Caractéristiques de la soudure } 2001 \text { dans les ménages }(n=579) \text { selon les zones de } \\
\text { risque }\end{array}$} \\
\hline & $\begin{array}{c}\text { Zone I } \\
\text { (risque maxi) }\end{array}$ & Zone II & Zone III & $\begin{array}{c}\text { Zone IV } \\
\text { (risque mini) }\end{array}$ & $\begin{array}{c}\text { Comparaison } \\
\text { statistique }\end{array}$ \\
\hline Nombre de mois d'anticipation & 3,0 & 1,8 & 2,8 & 2,3 & $\begin{array}{c}\mathrm{F}=3,9 \\
\mathrm{P}=0,009\end{array}$ \\
\hline $\begin{array}{l}\text { Achats céréaliers en \% des besoins } \\
\text { alimentaires annuels }\end{array}$ & 46,9 & 53,0 & 51,3 & 38,7 & $\begin{array}{l}\mathrm{F}=3,1 \\
\mathrm{P}=0,02\end{array}$ \\
\hline Nombre de jours de soudure & 175 & 210 & 180 & 157 & $\begin{array}{c}\mathrm{F}=7,9 \\
\mathrm{P}<0,0001\end{array}$ \\
\hline Yorouba / jour / équivalent-adulte & 0,27 & 0,23 & 0,25 & 0,25 & $\begin{array}{l}\mathrm{F}=3,8 \\
\mathrm{P}=0,01\end{array}$ \\
\hline $\begin{array}{l}\text { Revenu annuel net moyen* } \\
\text { par ménage en FCFA }\end{array}$ & 226.373 & 98.379 & 142.061 & 155.364 & $\begin{array}{c}\mathrm{F}=7,1 \\
\mathrm{P}<0,0001\end{array}$ \\
\hline $\begin{array}{l}\text { Source: enquêtes IRD/Univ. Ouagado } \\
\text { ransformation logarithmique de la variabl }\end{array}$ & EDES-Paris I, & pre-déc & 001.* $*$ & mparaison est & effectuée sur la \\
\hline
\end{tabular}

Basic characteristics of seasonal food shortage within apparent risk areas

En dépit d'une gestion prudente des stocks disponibles, l'amenuisement des disponibilités céréalières mobilisables pour la ration alimentaire de base a été précoce puisque $40 \%$ des ménages interrogés avaient déjà des greniers vides au mois de mars (milieu de saison sèche). Toutefois, si les déficits ont été importants dans toutes les zones de risques, des

\footnotetext{
${ }^{6}$ Cette mesure de base pour la vente des céréales et la préparation familiale équivaut à 2,7 à $3,0 \mathrm{~kg}$ de grain selon la technique manuelle de remplissage et la qualité du grain.
} 
disparités fortes ont été relevées d'une zone à l'autre (cf. tableau 1). Par ailleurs, ces valeurs moyennes masquent une forte variabilité de durée à l'intérieur de chaque zone, aussi bien entre les différents villages qu'entre cours collectives ou entre ménages.

L'étude des conditions de la soudure alimentaire selon les différentes zones montre que la zone de risque maximal est celle où l'anticipation a été la plus précoce $(\mathrm{p}=0,009)$. Par anticipation, il faut entendre toute opération d'achat de mil ou de sorgho réalisée avant la fin d'utilisation des greniers, soit consommé immédiatement, soit mis en réserve. Étant donné la dégradation des termes de l'échange entre bétail et céréales au cours de l'année (de l'ordre de 1 à 3), plus les achats sont précoces, moins ils sont coûteux et permettront au chef de ménage de réaliser de substantielles économies. Ces achats céréaliers en gros (par sac) ou au détail (en yorouba) ont été calculés sur la base des déclarations successives des chefs de ménage. La valeur moyenne du yorouba a été uniformisée à $3 \mathrm{~kg}$ pour les besoins de l'enquête mais reste susceptible de légères variations en fonction de la nature des récipients utilisés et de leur niveau de remplissage.

Cette zone I est également celle où les disponibilités céréalières finales, au cours de la soudure, ont été les plus importantes $(\mathrm{p}=0,01)$ alors même que les indicateurs secondaires apparents (saturation, densités, qualité des sols) sont dégradés. C'est aussi celle où les ménages disposaient des revenus moyens les plus élevés, une fois défalquées les ressources monétaires issues de la vente du bétail. Cette «épargne sur pied »constitue, en effet, le principal type de recours en situation de pénurie transitoire (Turner 2002): elle fournissait $34 \%$ des achats totaux en 2001. La validité de ces mesures a été confortée par l'ensemble des données collectées au cours de l'année 2002: la hiérarchie entre les zones de risque était préservée.

\begin{tabular}{|c|c|c|c|c|c|c|}
\hline \multicolumn{7}{|c|}{ Tableau 2} \\
\hline \multicolumn{7}{|c|}{$\begin{array}{l}\text { Caractéristiques socio-démographiques des } \\
\text { collectives }(n=180) \text { selon les zones de risque }\end{array}$} \\
\hline & & $\begin{array}{c}\text { Zone I } \\
\text { (risque maxi) }\end{array}$ & Zone II & Zone III & $\begin{array}{c}\text { Zone IV } \\
\text { (risque mini) }\end{array}$ & $\begin{array}{c}\text { Comparaison } \\
\text { statistique }\end{array}$ \\
\hline \multicolumn{2}{|c|}{ Nombre de ménages par cour } & 3,7 & 3,5 & 3,0 & 2,6 & $\begin{array}{c}\mathrm{F}=1,3 \\
\mathrm{P}=0,27\end{array}$ \\
\hline \multicolumn{2}{|c|}{ Nombre de sujets par cour } & 36,5 & 30,5 & 28,2 & 26,4 & $\begin{array}{l}\mathrm{F}=1,0 \\
\mathrm{P}=0,41\end{array}$ \\
\hline $\begin{array}{l}\text { Ethnie du CM : } \\
\text { (en \%) }\end{array}$ & $\begin{array}{l}\text { - gourmantché } \\
\text { - peuhl } \\
\text { - mossi }\end{array}$ & $\begin{array}{c}88,5 \\
2,5 \\
9,0\end{array}$ & $\begin{array}{c}92,9 \\
6,6 \\
0,6 \\
\end{array}$ & $\begin{array}{c}76,0 \\
21,6 \\
2,4\end{array}$ & $\begin{array}{c}69,8 \\
27,9 \\
2,3 \\
\end{array}$ & $\begin{array}{l}\text { Chi2=76,7 } \\
P<0,0001\end{array}$ \\
\hline \multicolumn{2}{|c|}{$\begin{array}{l}\% \text { de chefs de Ménage résidents } \\
\text { anciens (naissance ou > } 50 \text { ans) }\end{array}$} & 92,5 & 74,9 & 71,7 & 85,9 & $\begin{array}{l}\text { Chi2=30,2 } \\
\mathrm{P}<0,0001\end{array}$ \\
\hline \multicolumn{2}{|c|}{$\begin{array}{ll}\text { Niveau patrimoine* }: & - \text { «riche » } \\
(\text { en } \%) & -\ll \text { moyen } » \\
& - \text { «pauvre » }\end{array}$} & $\begin{array}{l}38 \\
42 \\
20\end{array}$ & $\begin{array}{l}22 \\
37 \\
41\end{array}$ & $\begin{array}{l}40 \\
24 \\
36\end{array}$ & $\begin{array}{l}35 \\
36 \\
29\end{array}$ & $\begin{array}{l}\text { Chi2=27,4 } \\
\mathrm{P}<0,0001\end{array}$ \\
\hline \multicolumn{2}{|c|}{$\%$ de ménages polygames } & 47,5 & 53,6 & 44,0 & 60,5 & $\begin{array}{c}\text { Chi2 }=6,9 \\
P=0,08\end{array}$ \\
\hline
\end{tabular}

Source : enquêtes IRD/Univ. Ouagadougou/IEDES-Paris I, novembre-décembre 2001.

* Indice calculé à partir de la qualité du logement, des biens de consommation courante, de la possession de bovins.

Socio-demographic characteristics of households and collective dwelling units (or compounds) within apparent risk areas

Les quatre zones diffèrent également selon leurs caractéristiques socio-démographiques (tableau 2). La zone I est notamment celle où le niveau des revenus nets et les patrimoines sont les plus élevés et où les ménages sont installés depuis le plus longtemps. La charge 
démographique par cour rurale tend également à être plus importante de même que le nombre d'unités de consommation. Par ailleurs, on voit très clairement que les ménages peuls sont beaucoup plus nombreux à être installés dans les zones III et IV où les potentialités pastorales restent encore importantes et où la compétition pour l'accès aux ressources est moindre.

\section{Les relations entre disponibilités et situation nutritionnelle}

En un deuxième temps, nous avons cherché à mettre en relation ces données de vulnérabilité avec les différents indicateurs nutritionnels. Seuls les indices nutritionnels de déficit énergétique et les indicateurs de maigreur correspondants (calculés selon les seuils recommandés), davantage susceptibles d'être modifiés par les variations saisonnières des disponibilités, aussi bien après récolte que pendant la période de la pénurie, sont analysés ici : indice poids-taille (P-T, en z-scores) de 0 à 9 ans, indice de masse corporelle (IMC, en $\mathrm{kg} / \mathrm{m}^{2}$ ) chez les adultes et IMC pour l'âge, de 10 à 20 ans. L'analyse statistique utilise le modèle linéaire pour les indices continus et le modèle logistique pour les indicateurs de maigreur.

\begin{tabular}{|c|c|c|c|c|c|c|}
\hline Tableau 3 & & & & & & \\
\hline Indices nutritionnel & de d & it énergéti & selon & ones de & que & \\
\hline & $\mathrm{n}$ & $\begin{array}{c}\text { Zone I } \\
\text { (risque maxi) }\end{array}$ & Zone II & Zone III & $\begin{array}{c}\text { Zone IV } \\
\text { (risque mini) }\end{array}$ & $\begin{array}{c}\text { Comparaison } \\
\text { statistique* }^{*}\end{array}$ \\
\hline $\begin{array}{l}\text { Indice poids-taille moyen des } \\
\text { enfants [0-5[ ans (en z- } \\
\text { scores) }\end{array}$ & 920 & $-0,51$ & $-0,74$ & $-0,72$ & $-0,79$ & $\begin{array}{c}\mathrm{F}=4,3 \\
\mathrm{P}=0,005\end{array}$ \\
\hline $\begin{array}{c}\text { Indice poids-taille moyen des } \\
\text { enfants [5-10[ ans (en z- } \\
\text { scores) }\end{array}$ & 840 & $-0,37$ & $-0,55$ & $-0,70$ & $-0,75$ & $\begin{array}{c}\mathrm{F}=10,7 \\
\mathrm{P}<0,0001\end{array}$ \\
\hline $\begin{array}{c}\text { IMC moyen }\left(\mathrm{kg} / \mathrm{m}^{2}\right) \text { des } \\
\text { garçons de }[10-21[\text { ans }\end{array}$ & 372 & 17,71 & 17,38 & 16,86 & 17,54 & $\begin{array}{l}\mathrm{F}=3,7 \\
\mathrm{P}=0,01\end{array}$ \\
\hline $\begin{array}{l}\text { IMC moyen }\left(\mathrm{kg} / \mathrm{m}^{2}\right) \text { des } \\
\text { filles de }[10-21[\text { ans }\end{array}$ & 507 & 18,20 & 17,87 & 17,55 & 17,23 & $\begin{array}{c}\mathrm{F}=6,3 \\
\mathrm{P}=0,0003\end{array}$ \\
\hline $\begin{array}{l}\text { IMC moyen }\left(\mathrm{kg} / \mathrm{m}^{2}\right) \text { des } \\
\text { adultes hommes }\end{array}$ & 651 & 21,51 & 20,91 & 21,28 & 20,93 & $\begin{array}{l}\mathrm{F}=3,7 \\
\mathrm{P}=0,01\end{array}$ \\
\hline $\begin{array}{l}\text { IMC moyen }\left(\mathrm{kg} / \mathrm{m}^{2}\right) \text { des } \\
\text { adultes femmes }\end{array}$ & 749 & 21,33 & 20,41 & 20,24 & 20,50 & $\begin{array}{c}F=9,8 \\
P<0,0001\end{array}$ \\
\hline
\end{tabular}

Energy deficiency rates within apparent risk areas

En fonction de la zone géographique de résidence, l'analyse brute de l'état nutritionnel des individus, apprécié ici à travers les indices de déficit énergétique ajustés sur l'âge et le sexe, fait apparaître un niveau de malnutrition systématiquement plus faible dans la zone de risque apparent maximal (cf. tableau 3) : meilleur indice P-T moyen chez les moins de 5 ans $(\mathrm{p}=0,005)$ comme chez les 5-9 ans $(\mathrm{p}<0,0001)$, IMC moyen plus élevé chez les adolescents garçons $(p=0,01)$ et filles $(p=0,0003)$, ainsi que chez les adultes hommes $(p=0,01)$ comme femmes $(\mathrm{p}<0,0001)$. $\mathrm{Si}$, au lieu des indices moyens on étudie les pourcentages de maigreur, la tendance est identique mais la différence n'est significative que chez les adolescentes $(\mathrm{p}=0,006)$ et chez les femmes $(\mathrm{p}=0,004)$. Les trois autres zones se distinguent moins bien les unes des autres et, selon les catégories d'âge et de sexe, la malnutrition peut apparaître plus prononcée dans la zone de risque apparent minimal ou dans une des deux zones intermédiaires. 
Compte tenu du fait que les zones de risque apparent se distinguent les unes des autres par les caractéristiques de la soudure et par un certain nombre de traits socio-démographiques, une analyse multivariée a été effectuée, en deux temps, pour évaluer le rôle éventuel de ces différences entre zones sur la répartition de la malnutrition (tableau 4).

On a, tout d'abord, introduit dans les modèles les variables caractérisant la soudure. On a alors constaté que les résultats bruts étaient relativement peu modifiés, ce qui tient au fait que les conditions de la soudure ne se sont pas révélées, ici et de la façon dont elle sont été estimées, des déterminants importants des indices nutritionnels.

\begin{tabular}{|c|c|c|c|c|c|c|c|}
\hline \multicolumn{8}{|l|}{ Tableau 4} \\
\hline \multicolumn{8}{|c|}{$\begin{array}{l}\text { Indices nutritionnels de déficit énergétique selon les zones de risque : comparaisons } \\
\text { ajustées sur les caractéristiques de la soudure* et sur les caractéristiques socio- } \\
\text { démographiques } * *\end{array}$} \\
\hline & $\begin{array}{c}\text { Type d' } \\
\text { ajustement }\end{array}$ & $\mathrm{n}$ & $\begin{array}{c}\text { Zone I } \\
\text { (risque maxi) }\end{array}$ & Zone II & Zone III & $\begin{array}{c}\text { Zone IV } \\
\text { (risque mini) }\end{array}$ & $\begin{array}{l}\text { Comparaison } \\
\text { statistique* }^{*}\end{array}$ \\
\hline \multirow{2}{*}{$\begin{array}{l}\text { Indice poids-taille } \\
\text { moyen des enfants } \\
{\left[0-5\left[\text { ans (en } \mathrm{Z}^{-}\right.\right.} \\
\text {scores) }\end{array}$} & Soudure & 816 & $-0,52$ & $-0,71$ & $-0,75$ & $-0,81$ & $\begin{array}{l}\mathrm{F}=3,3 \\
\mathrm{P}=0,02\end{array}$ \\
\hline & $\begin{array}{c}\text { Soudure + } \\
\text { socio-démo }\end{array}$ & 750 & $-0,54$ & $-0,69$ & $-0,72$ & $-0,74$ & $\begin{array}{l}F=1,5 \\
P=0,21\end{array}$ \\
\hline \multirow{2}{*}{$\begin{array}{l}\text { Indice poids-taille } \\
\text { moyen des enfants } \\
{[5-10[\text { ans (en z- }} \\
\text { scores) }\end{array}$} & Soudure & 766 & $-0,33$ & $-0,55$ & $-0,68$ & $-0,63$ & $\begin{array}{c}\mathrm{F}=7,8 \\
\mathrm{P}<0,0001\end{array}$ \\
\hline & $\begin{array}{c}\text { Soudure + } \\
\text { socio-démo }\end{array}$ & 709 & $-0,39$ & $-0,61$ & $-0,69$ & $-0,58$ & $\begin{array}{c}\mathrm{F}=4,3 \\
\mathrm{P}=0,005\end{array}$ \\
\hline \multirow{2}{*}{$\begin{array}{l}\text { IMC moyen }\left(\mathrm{kg} / \mathrm{m}^{2}\right) \\
\text { des garçons de }[10- \\
21[\text { ans }\end{array}$} & Soudure & 344 & 17,67 & 17,32 & 16,67 & 17,49 & $\begin{array}{c}\mathrm{F}=4,6 \\
\mathrm{P}=0,004\end{array}$ \\
\hline & $\begin{array}{l}\text { Soudure + } \\
\text { socio-démo }\end{array}$ & 314 & 16,82 & 16,32 & 15,84 & 16,91 & $\begin{array}{l}F=3,5 \\
P=0,02\end{array}$ \\
\hline \multirow{2}{*}{$\begin{array}{l}\text { IMC moyen }\left(\mathrm{kg} / \mathrm{m}^{2}\right) \\
\text { des filles de }[10-21[ \\
\text { ans }\end{array}$} & Soudure & 461 & 18,22 & 17,91 & 17,55 & 17,19 & $\begin{array}{c}\mathrm{F}=6,1 \\
\mathrm{P}=0,0004\end{array}$ \\
\hline & $\begin{array}{c}\text { Soudure + } \\
\text { socio-démo }\end{array}$ & 416 & 18,30 & 18,09 & 17,55 & 17,58 & $\begin{array}{l}\mathrm{F}=3,1 \\
\mathrm{P}=0,03\end{array}$ \\
\hline \multirow{2}{*}{$\begin{array}{l}\text { IMC moyen }\left(\mathrm{kg} / \mathrm{m}^{2}\right) \\
\text { des adultes hommes }\end{array}$} & Soudure & 588 & 21,60 & 21,10 & 21,42 & 21,17 & $\begin{array}{c}\mathrm{F}=2,0 \\
\mathrm{P}=0,12\end{array}$ \\
\hline & $\begin{array}{c}\text { Soudure + } \\
\text { socio-démo }\end{array}$ & 536 & 21,13 & 20,55 & 20,92 & 21,02 & $\begin{array}{l}\mathrm{F}=1,9 \\
\mathrm{P}=0,12\end{array}$ \\
\hline \multirow{2}{*}{$\begin{array}{l}\text { IMC moyen }\left(\mathrm{kg} / \mathrm{m}^{2}\right) \\
\text { des adultes femmes }\end{array}$} & Soudure & 687 & 21,32 & 20,60 & 20,16 & 20,44 & $\begin{array}{c}\mathrm{F}=8,0 \\
\mathrm{P}<0,0001\end{array}$ \\
\hline & $\begin{array}{c}\text { Soudure + } \\
\text { socio-démo }\end{array}$ & 626 & 21,23 & 20,72 & 20,19 & 20,45 & $\begin{array}{c}\mathrm{F}=4,7 \\
\mathrm{P}=0,003\end{array}$ \\
\hline
\end{tabular}

Source : enquêtes IRD/Univ. Ouagadougou/IEDES-Paris I, janvier-mars 2002.

* avec ajustement sur l'âge et sur le sexe, s'il y a lieu et + variables de soudure (revenus, vente de bétail, anticipation, durée, disponibilités alimentaires moyennes).

** ajustement sur toutes les variables ci-dessus + taille de la cour, ethnie, religion, niveau de patrimoine, niveau d'hygiène, ancienneté de résidence.

Energy deficiency rates within apparent risk areas : comparisons adjusted on seasonal food shortage and socio-demographic characteristics

Dans un second temps, les comparaisons ont été ajustées, en plus des caractéristiques de soudure, sur un certain nombre de variables socio-démographiques. La zone de risque maximal reste alors globalement celle où l'état nutritionnel est meilleur, mais la relation initiale s'estompe nettement : elle reste néanmoins significative chez les enfants de 5-9 ans $(\mathrm{p}=0,005)$, les adolescents garçons $(\mathrm{p}=0,02)$ et filles $(\mathrm{p}=0,03)$ et les femmes $(\mathrm{p}=0,008)$. 
Une partie des différences observées dans l'analyse brute est donc attribuable à des différences entre les zones selon des caractéristiques comme l'ethnie, le niveau de patrimoine ou d'hygiène dans les ménages. Mais la relation n'étant globalement pas remise en cause, d'autres facteurs doivent être recherchés pour expliquer ce paradoxe d'une situation nutritionnelle meilleure dans la zone de risque apparent maximal.

\section{Les enseignements méthodologiques pour l'action}

Le niveau d'exposition des ménages ruraux de la Gnagna au risque d'insécurité semble donc, de prime abord, dépendre du niveau des stocks disponibles sur l'exploitation - faute de pouvoir anticiper, d'une année à l'autre, d'éventuels déficits alimentaires. Et ce d'autant plus que la saison des pluies se combine souvent avec un enclavement géographique qui compromet fortement l'accessibilité aux marchés physiques. Cependant, même à l'échelle du ménage rural, cet indicateur de base reste difficile à mesurer étant donné la multiplicité des lieux de stockage (concession rurale, champs et hameaux de culture plus éloignés). Par conséquent, on peut légitimement poser la question de la fiabilité des bilans céréaliers régionalisés, réalisés à partir de données extrapolées de récoltes collectées sur un panel réduit d'exploitations témoins sur lesquelles s'appuient généralement les interventions sectorielles.

De manière plus fondamentale, cette analyse montre que le niveau de risque apparent, basé sur les contraintes environnementales au sens large, est loin de correspondre au niveau réel des disponibilités céréalières familiales. De plus, des variations importantes des stocks céréaliers ont été observés d'une année sur l'autre, confirmant la nécessité de reconduire des enquêtes sur plusieurs années agricoles. Par ailleurs, pour évaluer ce risque d'insécurité alimentaire, il importe également de prendre en compte, le volume important d'achats céréaliers réalisé au cours de la période de soudure alimentaire. En outre, une place centrale doit être accordée, en zone sahélienne et sahélo-soudanienne, aux différents types d'élevage pratiqués pour leur contribution stratégique en période de soudure (d'Aquino 2000), puisque le cheptel est essentiellement destiné à être revendu pour acheter des céréales traditionnelles locales (CILSS et al. 2000). Enfin, on ne doit pas négliger les mécanismes de redistribution communautaire au sein de chaque unité spatiale d'habitat et qui s'expriment par des échange de plats, des préparations alimentaires collectives, ou des formes plus importantes de prise en charge pour des ménages en difficulté (immigrants récents par exemple). Ces différents éléments sont, certes, difficiles à collecter mais s'avèrent indispensables dans le cadre d'une analyse approfondie des relations entre insécurité alimentaire et malnutrition.

Ce texte montre également que le lien entre disponibilités céréalières et état nutritionnel des individus n'est pas évident. Les conditions de la soudure alimentaire, telles qu'appréhendées ici, semblent ainsi jouer un rôle moins important vis-à-vis de l'état nutritionnel que les caractéristiques d'ordre socio-démographique (religion, statut, niveau d'éducation, ethnie) et économique (revenus). Tout ceci suggère que, confrontés à l'irrégularité de la production céréalière familiale et à la récurrence de la disette alimentaire, les ménages les plus exposés ont progressivement développé des stratégies d'adaptation à relativement long terme et d'anticipation saisonnière (Marchal 1990), permettant de gommer partiellement les aléas conjoncturels.

Enfin, un des enseignements de cette recherche qui rejoint, de manière implicite, l'insatisfaction exprimée par les donateurs privés et institutionnels, est que l'identification des groupes vulnérables et des malnutris comme le ciblage de poches de vulnérabilité restent très difficiles à mettre en œuvre, dans un contexte d'information fragmentaire et d'instabilité géographique des populations rurales, pour la mise en place de politiques cohérentes et pour l'intervention. Cette identification est rendue encore plus aléatoire par les retournements des 
indicateurs en fonction de l'échelle géographique et statistique d'analyse. Il importe donc à la fois de s'affranchir progressivement du zonage géographique initial (selon l'intensité des risques apparents) tout en systématisant les analyses permettant de mettre en évidence la complexité organisationnelle et fonctionnelle de chaque communauté familiale en situation d'insécurité alimentaire.

Ainsi, ce sont bien les modes de gestion temporelle des ressources alimentaires et la capacité à acquérir des aliments sur le marché ou à reconstituer une «épargne sur pied » (bétail) qui conditionnent le niveau de vulnérabilité réelle de chaque ménage rural dans un environnement incertain et instable (Bebbington 1999). Parallèlement, les modalités d'allocation des ressources alimentaires - et partant les inégalités individuelles d'accès comme les modèles de comportement alimentaire semblent exercer une influence forte sur l'état nutritionnel des individus.

\section{Références}

D’Aquino, Patrick. 2000. «L'agropastoralisme au nord du Burkina (province du Soum) : une évolution remarquable mais encore inachevé. » Paris IRD : Autrepart $15: 29-47$.

Bebbington, A. 1999. "Capitals and Capacities : a Framework for Analyzing Peasant Viability, Rural Livelihoods and Poverty." World Development 27 (12) : 2021-2044.

Clay, Daniel. C., Molla Daniel. and Habtewold, Debebe. 1999. "Food Aid Targeting in Ethiopia. A Study of Who Needs it and Who Gets it." Food Policy 24 : 391-409.

CILSS. 2000. Le contexte de la vulnérabilité structurelle par système de production au Burkina Faso, Niger, Mali et Sénégal, octobre 2000, Centre régional Agrhymet, 27 p.

CILSS, OMM et Coopération Italienne, 2000. Approche méthodologique pour l'intégration de l'élevage dans la caractérisation des zones d'insécurité alimentaire structurelle au Burkina Faso-Mali-Niger-Sénégal, Rencontre régionale sur le «Suivi de la Situation Alimentaire au Sahel et Préparatifs de la Campagne Agricole 2000-2001 (Niamey 26 - 30 juin 2000), 11 p.

Courade, Georges et al. 2001. "Paupérisation et inégalités d'accès aux ressources ». In Inégalités et politiques publiques en Afrique. Pluralité des normes et jeux d'acteurs, édité par Gérard Winter. Paris : Karthala, Col. Economie et développement : 25-39.

Courade, Georges. 1996. «La banalisation de l'insécurité alimentaire. » In Alimentation et agriculture durables, Paris, Aménagement et Nature 123 : 73-80.

Dilley, M. and Boudreau, T. E., 2001. "Coming to Terms with Vulnerability : a Critique of the Food Security Definition." Food Policy 26 : 229-247.

Eele, G. 1994. "Indicators for Food Security and Nutrition Monitoring : a review of experience from Southern Africa." Food Policy 19 (3) : 314-328.

Egg, Johnny. et Gabas Jean-Jacques. 1997. «La prévention des crises alimentaires au Sahel et le rôle des dispositifs d'information. » Paris : Stateco 87-88 : 5-20.

Eldin, Michel. 1989. "Du risque de sécheresse au risque de mauvaise récolte : un effort de clarification des concepts. » pp. 19-23, In Le risque en agriculture. Paris : ORSTOM, Col. A travers champs : 19-23.

Gado, B. A. 1993. Une histoire des famines au Sahel : études des grandes crises alimentaires (XIX ${ }^{\circ}-$ $X X^{\circ}$ siècles). Paris : L'Harmattan, Col. Racines du présent : $200 \mathrm{p}$.

Janin, Pierre. 2004. «Gestion spatio-temporelle de la soudure alimentaire dans le Sahel burkinabé. » Paris : PUF. Revue Tiers-Monde (à paraitre décembre 2004).

Janin, Pierre. et Martin-Prével, Yves. 2003. «Disponibilités alimentaires, niveaux de risque apparent et état nutritionnel en milieu rural sahélien burkinabè. » $2^{\text {ème }}$ Atelier International «Voies alimentaires d'amélioration des situations nutritionnelles en Afrique de l'Ouest : Le rôle des technologues alimentaires et des nutritionnistes »(23-28 Novembre 2003) Ouagadougou: Université de Ouagadougou-IRD-Université de Wageningen-FAO : $10 \mathrm{p}$.

Jaspers, S. and Shoham, J. 1999. "Targeting the Vulnerable : a Review of the Necessity and Feasability of Targeting Vulnerable Households." Disasters 23 (4) : 359-372. 
Jayne, T. S. Strauss, John. Yamano, Takashi. and Molla, Daniel 2001. "Giving to the poor ? Targeting of food aid in Ethiopia." World Development 29 (5) : 887-910.

Marchal, Jean-Yves. 1990. «En Afrique soudano-sahélienne : la course contre le temps. » In Le risque en agriculture, édité par Michel Eldin and Pierre Milleville. Paris : ORSTOM. Col. A travers champs : 225-267.

Moseley, W. G. and Logan, B. I. 2001. "Conceptualizing Hunger Dynamics : A Critical Examination of Two Famine Early Warning Methodologies in Zimbabwe.”, Applied Geography 21 : 223248.

Ouedraogo, (François de Charles. 2001. Atlas de la vulnérabilité alimentaire. IRD/Université de Ouagadougou : $150 \mathrm{p}$.

PAM, juin 1999. Evaluation de la sécurité alimentaire et nutritionnelle des populations rurales de la zone soudanienne au Tchad: $39 \mathrm{p}$.

PAM, juin 2002. Vulnerability Analysis and Mapping - Standard Analytical Framework. Role and Objectives of VAM Activities to Support WFP food-oriented Interventions : $11 \mathrm{p}$.

Reardon, Thomas. and Matlon, Peter. 1989. "Seasonal Food Insecurity and Vulnerability in Droughtaffected Regions of Burkina Faso." In Seasonal variation in the Third World agriculture, edited by D. Sahn. Baltimore : John Hopkins University Press : 118-136.

Sawadogo, Prosper. 2002. Les pratiques alimentaires dans la province de la Gnagna. Mémoire de DEA «Biologie, technologie alimentaire et nutrition humaine » : Université de Ouagadougou.

Turner, Matthew. D. and Williams, Thimothy. O. 2002. "Livestock Market Dynamics and Local Vulnerabilities in the Sahel." World Development 30 (4) : 683-705.

UNICEF, 1999. The State of the World's Children 1999. Education. New York : UNICEF : $134 \mathrm{p}$.

WHO, 1995. Physical Status : the Use and Interpretation of Anthropometry. WHO Technical Report Series 854. Geneva.

Watts, M. \& Bohle, H. 1993. "The Space of Vulnerability : the Causal Structure of Hunger and Famine.” Progress in Human Geography 17 (1) : pp. 43-67. 